\title{
Research and Development in HPGe detectors at LNL
}

M. Gelain, G. Maggioni, S. Carturan and D. De Salvador

University of Padova and INFN Laboratori Nazionali di Legnaro, Italy

D.R. Napoli*, M. Loriggiola, D. Rosso and P. Cocconi

INFN, Laboratori Nazionali di Legnaro

E-mail: hapolidelnl.infn.it

G. Della Mea

University of Trento and INFN Laboratori Nazionali di Legnaro, Italy

\section{J. Eberth}

Institut für Kernphysik, University of Cologne, Germany

\section{G. Mariotto, A. De Lorenzi and G. Valotto}

University of Verona and INFN Laboratori Nazionali di Legnaro, Italy

\section{M.G. Grimaldi and S. Tati}

University of Catania, Italy

\begin{abstract}
High-purity Germanium (HPGe) detectors continue to be a fundamental tool in nuclear gamma spectroscopy. New techniques for the tracking of the gamma interactions inside the HPGe crystals, are opening a new era in the use of these detectors but still new R\&D is needed. In the present work we discuss results on the characterization of a new passivation technique studied in the framework of our research program in HPGe detector technologies.
\end{abstract}

X Latin American Symposium on Nuclear Physics and Applications (X LASNPA),

1-6 December 2013

Montevideo, Uruguay

\footnotetext{
* Speaker.
} 


\section{Introduction}

Germanium detectors have reached an unprecedented level of sophistication and they are still the best solution for high resolution gamma spectroscopy. On the other side, they are expensive, need specific support infrastructures also during normal operation and from time to time require expensive repairs. Recently advances in electronics and cryogenic cooling systems could help to reduce the standard operational costs of Ge detectors but technological R\&D is still needed to produce cheaper and most reliable detectors.

The Legnaro National Labs (LNL) has a long tradition in nuclear spectroscopy studies. Many gamma-ray arrays have been operated at LNL in the last 30 years since the installation of MIPAD in 1987 [四]. In 1991 we have designed and installed GASP, a gamma array composed of 40 Hyper Pure Ge detectors (HPGe), that have been run since 1992 [] till 2012. During the same period we have installed other gamma arrays at LNL in collaboration with institutes of all Europe, like EUROBALL [3, 田, 回] in 1997-1998, CLARA [可] from 2004 to 2008 and recently, after a short demonstration phase, we have performed the first physics campaign of AGATA in the period 2009

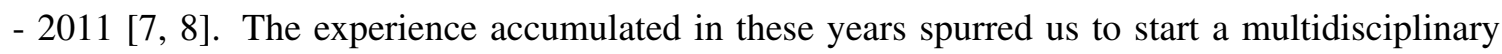
research program for developing new technologies for the fabrication of highly segmented HPGe detectors, useful for gamma tracking.

\section{Nuclear Spectroscopy and Ge Detector Technology}

Technological advances and basic research activities are intimately connected. It can be seen in Ref. [9, [0] how the experimental progress in nuclear spectroscopy is strongly related with the technological improvements in the manufacture of Ge detectors.

High resolution nuclear spectroscopy was born in the sixties with the introduction of the Ge(Li) detectors [], [2]. In the seventies High purity Germanium detectors (HPGe or hyperpure Ge detectors) [[13, [4]] became available with practical advantages that allowed the realization of multidetector systems in the eighties. These gamma detectors systems, frequently called as gamma-ray arrays (or shortly: gamma-arrays), increased the efficiency to correlate gamma-rays detected in different detectors within a small temporal window, called coincidence window. Gamma-gamma coincidences are of fundamental importance to build up complex level schemes. The granularity and efficiency of the gamma-ray arrays is closely related with the number of gammas that can be measured simultaneously in the same event (gamma coincidence fold of the event). The higher the granularity and efficiency of the array, the higher the gamma fold and the sensitivity of the array.

The next important step was done in the nineties with the introduction of crystal encapsulation by Eberth and Lieder in collaboration with EURISYS MEASURES, a private company born in Strasbourg, France, which invested important efforts in R\&D [प5]]. The encapsulation was a significant step to arrive to the crystal segmentation one year later [ए6].

The importance of the encapsulation concept is related with the protection of the HPGe crystal surfaces inside the cryostats. This protection is necessary when the number of electronic elements inside the cryostat increases, because it gives rise to an increase of the risk of contamination of these surfaces. 
The segmentation and the new digital electronics, together with the increasing calculation power of computers make possible the development of the gamma tracking concept [ए7], [18] that have opened a new era in the use of HPGe detectors.

In this short introduction we mention the most important steps forward in Ge detectors technology that allowed to improve the sensitivity of the gamma-arrays by almost 7 orders of magnitude in the last 50 years (see for example Figure 40 of Ref. [Q] ). But it is important to note that the last step, with the introduction of the gamma tracking concept and digital electronics, is responsible for the last factor 100 .

In the next section we will present preliminar results on the characterisation of a new passivation method we have proposed recently in the framework of a multidisciplinary R\&D project we are carrying on in Italy to improve the techniques for producing better and more reliable HPGe detectors.

\section{Passivation techniques in HPGe detectors}

The heart of a HPGe detector is, basically, an inverse polarized diode of high purify Ge crystal wherein the incoming gamma-rays will interact, and the resulting electron-hole pairs will be collected by two contacts placed on the opposite sides of the crystal. For other details please see Ref. [10].

From the point of view of the raw material, Germanium crystal manufacturers reached good standards in the growth of large volume crystals of hyper pure Ge, i.e. with a low level of impurity concentration (around $10^{10}$ atoms $/ \mathrm{cm}^{3}$ ). In particular, we have been working in the last years with $\mathrm{n}$-type detectors that have a better response to neutron damage recovering and a thinner external contact layer as compared to the p-type detectors.

The most critical point in the HPGe detector manufacturing is the treatment and care of the passivated crystal surface that has to hold the high voltage applied in between the contacts during the operation of the detector. Unlike Silicon, Germanium does not have a stable oxide and it is necessary to apply on it a passivation layer [ [20]]. As far as we can find in literature [Q], commercial passivation is obtained by deposition processes like $\mathrm{SiO}_{2}$ or amorphous $\mathrm{Ge}$ sputtering, but these passivation layers induce a dead layer near the surface that reduces the efficiency of charge collection [Q]. The dead layer creates a new deep level that promotes the recombination of the electron-hole pairs. As a consequence, the resolution of the gamma spectra, acquired near the surface, is of unpredictable quality. A good example is shown by Eberth and Simpson in Ref. [Q] where they present some examples of spectra acquired in different positions of the passivated surface of a commercial detector as well as the measured dead layer profile near the passivated surface (Figure 4 and 5 of that reference).

The first difficulty in the R\&D on passivation techniques for HPGe detectors is that many of the methods studied for semiconductor manufacturing cannot be applied to HPGe detectors whose passivated surface has to sustain a potential gradient of about $1 \mathrm{KV} / \mathrm{cm}$ and has to be stable in thermal and vacuum cycles that go from cryogenic till annealing temperatures (around $100^{\circ} \mathrm{C}$ ). The second difficulty is that many properties of the passivated layer can be studied on Ge wafers in laboratory but the conclusive test can be done only on a real detector with gamma-rays. 
After studying the bulk characteristics of the detector with calibrated gamma sources, the scanning of the passivated surface with low energy gamma-rays allows to characterize the quality of the passivation, the charge collection in the proximity of the passivated surface when different bias voltages are applied and the influence of the permanent trapping on the signal formation.

\section{Experimental Results}

Planar HPGe cylindrical detectors for these tests were produced with a contact of implanted $\mathrm{B}$ in one of the planar faces of the cylinder and a diffused Li contact in the opposite side. On the lateral surface a methoxide-terminated passivation has been created [2]]. This passivation shows good electrical properties but is not stable at all, and needs to be coated by a protective thin layer that we have developed at LNL and is under patent [[22].

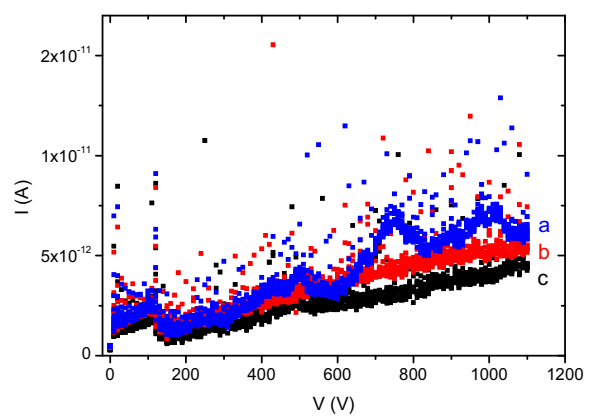

Figure 1: Inverse current of the crystal as a function of the applied voltage: (a) after the passivation, (b) after the protective coating deposition and (c) after a thermal cycle with annealing.

To determine the quality of the Ge diode, we have measured the inverse current as a function of the applied voltage (Fig. W): (a) after the passivation, (b) after the protective coating deposition and (c) after a thermal cycle with annealing. As can be seen the coating does not compromise the insulation of the detector. In order to evaluate the stability of the protective coating, the test has been repeated one and two years later and after repeated thermal cycles.

\subsection{Experimental Setup}

We have tested a cylindrical planar HPGe detector of $2.1 \mathrm{~cm}$ (height) x $3.9 \mathrm{~cm}$ (diameter) made as described above. The crystal has been mounted and cooled at $77 \mathrm{~K}$ inside a commercial cryostat
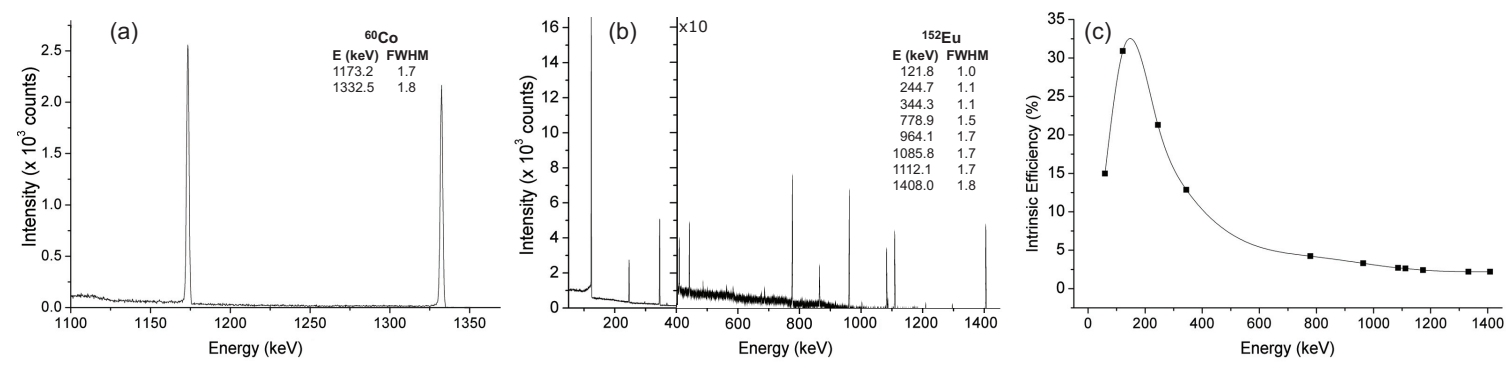

Figure 2: Spectra of ${ }^{60} \mathrm{Co}$ (a) and ${ }^{152} \mathrm{Eu}$ (b) taken with our detector. The intrinsic efficiency of the HPGe detector is shown in (c) as a function of energy (experimental points are interpolated with a line). 
which has been modified to reduce the amount of material in between the crystal and the source in order to allow also the scanning of the lateral surface from one contact to the other.

We have operated standard NIM electronics and acquired the spectra with a shaping time of 3 microseconds. Typical ${ }^{152} \mathrm{Eu}$ and ${ }^{60} \mathrm{Co}$ calibration sources spectra can be seen in Fig. \.

An intrinsic efficiency of $30 \%$ at $121.78 \mathrm{keV}$ and a FWHM $=1.87 \mathrm{keV}$ at $1332.50 \mathrm{keV}$, as shown in Fig. [, were measured for this detector. The efficiency was calculated by using the photopeak integral normalized to the source activity, the active volume of the detector, the solid angle, the live time of the acquisition and the branching ratio of the source.

\subsection{Measurement and Interpretation}

For the characterization of the passivated surface, we have scanned it with a collimated ${ }^{241} \mathrm{Am}$ gamma-calibration source. As can be seen in the simplified scheme shown in Fig. [1, the collimator consists in a $2.5 \mathrm{~cm}$ thick lead block with a hole of $1.2 \mathrm{~mm}$ of diameter placed at $9.6 \mathrm{~mm}$ far from the crystal. The collimator can be translated along the detector surface at almost any angle. We have taken the Li contact position as reference for the displacement measurements.

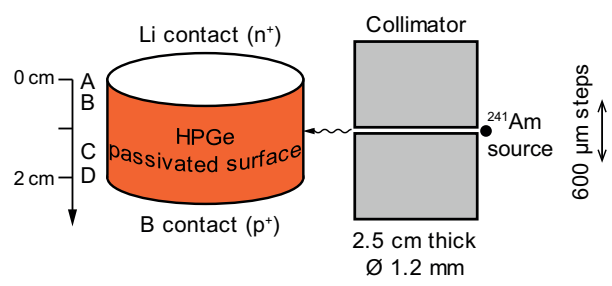

Figure 3: Simplified scheme of the scanning setup. The collimator and the ${ }^{241} \mathrm{Am}$ source can move along the passivated side of the crystal (in red) from the top ( $\mathrm{Li}$ contact), to the opposite B contact (at $2 \mathrm{~cm}$ ) with $0.6 \mathrm{~mm}$ steps.

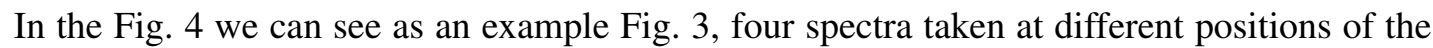
passivated surface as shown in Fig. [3. To determine the efficiency of the charge collection we have measured the photo-peak area normalized to the acquisition live time. In Fig. 1 we see the counting rate in the photo-peak (cps) as a function of the position at $0.6 \mathrm{~mm}$ steps. The error in the longitudinal position was given by the size of the gamma-ray spot that we estimated in $1.8 \mathrm{~mm}$. The measurements have been repeated at 3 bias voltages and in the figure it is possible to observe the effect of the voltage on the surface charge collection. The full depletion of the detector was reached around $2000 \mathrm{~V}$ and we decided to operate it at $2500 \mathrm{~V}$. Using the Lambert-Beer law [एT], we have determined, from the curve at $2500 \mathrm{~V}$, an average dead layer thickness of about $0.5 \mathrm{~mm}$ with a maximum of $3 \mathrm{~mm}$ near the contacts, that is much less than the value measured in Ref. [Q] for a commercial detector. Moreover, the thickness of the dead layer is much more regular than that of the commercial passivation.

As discussed in Ref. [Q], commercial passivation techniques could be responsible of an incomplete charge collection near the passivated surface of the detector (see for example Figure 4 in Ref. [Q]. Our passivation technique is not showing that effect and, as can be seen in Fig. 团, the ${ }^{241} \mathrm{Am}$ spectra taken in different positions along the surface of the crystal are well shaped. All the spectra were taken at constant photo-peak area, then the observed increasing of the background when approaching the B contact, is related with different dynamics in electron-hole recombination 

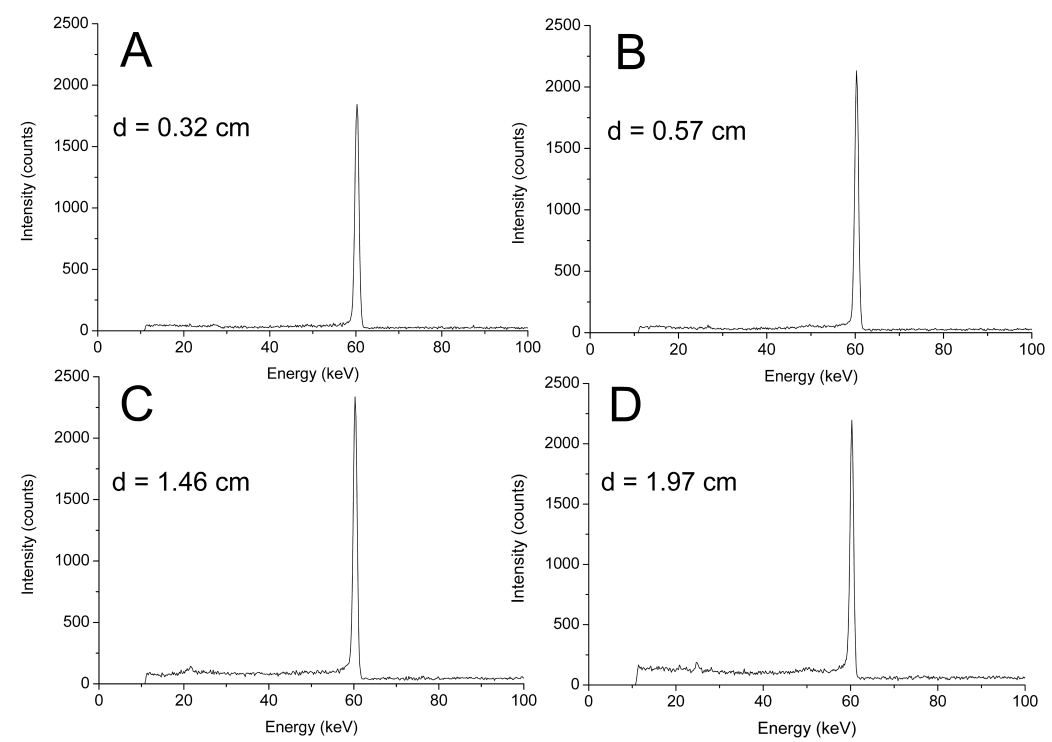

Figure 4: ${ }^{241} \mathrm{Am}$ gamma-ray spectra taken, with the detector polarized at $2500 \mathrm{~V}$, along the passivated surface at four different positions A, B, C and D represented in the scheme of Fig.3. The spectra are well shaped also quite near the contacts. As the photo-peak area is constant, we observe how the background increases when going from the Li contact (A) to the Boron (D) due to the dynamics of charge collection.

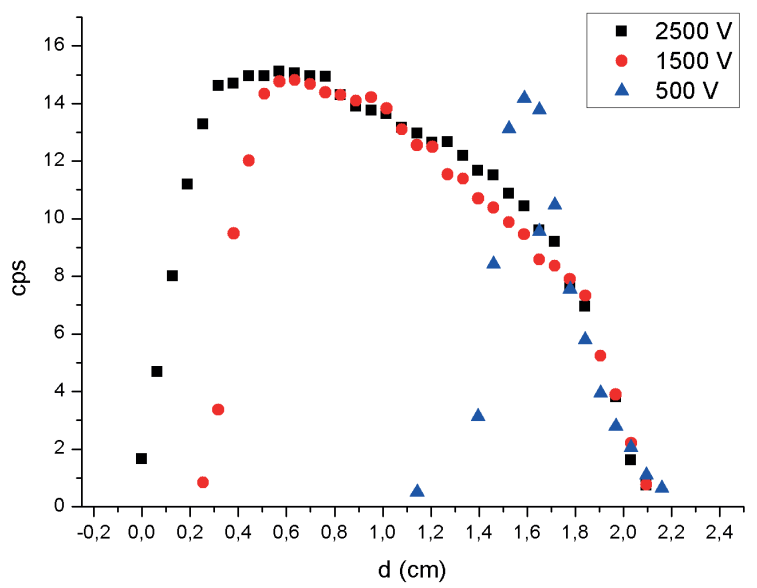

Figure 5: The figure shows the photo-peak area of the collimated ${ }^{241}$ Am source divided the acquisition live time (counts per second: cps) as a function of the position along the passivated surface of the HPGe detector, for three different polarization voltages. The position is taken from the Li contact as described in the text. It is possible to see how the increasing of the voltage improves the charge collection.

phenomena. A more detailed and quantitative analysis exceeds the scope of this presentation and will be published in a more extended work.

\section{Conclusions}

As advances in high resolution nuclear spectroscopy are closely related with technological improvements, we started a R\&D program in HPGe detectors technology aimed at achieving more 
reliable complex HPGe detectors. We have produced planar detectors, starting from commercial HPGe crystals, using a surface passivation method which shows good charge collection near the surface with minimum dead layers. To apply this passivation method we have implemented an innovative protection technique for Ge detectors that, not only can replace the crystal encapsulation of new devices but also can be used for any HPGe detector. The preliminary results obtained with this passivation method are promising if one takes into account its stability and its influence on the charge collection, which is better than the one obtained in commercial detectors.

\section{Acknowledgments}

This work was supported in part by the 7th Framework Program FP7/2007-2013, under grant agreement No. 262010 - ENSAR-INDESYS.

\section{References}

[1] G. Lobianco, Prog. Part. Nucl. Phys. 28 (1992) 487.

[2] D. Bazzacco et al., Phys. Lett. B 309 (1993) 235.

[3] J. Simpson, Zeit.fur Phys. A 358 (1997) 139.

[4] C. Rossi Alvarez, Nuovo Cim. Soc. Ital. di Fis. A, 111 (1998) 601.

[5] S. Tormanen et al., Phys. Lett. B 454 (1999) 8.

[6] A. Gadea et al., Eur. Phys. J. A 20 (2004) 193.

[7] A. Gadea et al., Nucl. Instr. and Meth. A 654 (2011) 88.

[8] S. Akkoyun et al., Nucl. Instr. and Meth. A 668 (2012) 26.

[9] J. Eberth and J. Simpson, Prog. Part. Nucl. Phys. 60,(2008) 283.

[10] K. Vetter, Annu. Rev. Nucl. Part. Sci. 57 (2007) 363.

[11] E.M. Pell, J. Appl. Phys. 31 (1960) 291.

[12] D.V. Freck, J. Wakefield, Nature 193 (1960) 669.

[13] R.N. Hall, T.J. Soltys, IEEE Trans. Nucl. Sci NS-18 (1971) 160.

[14] W.L. Hansen, Nucl. Instr. and Meth. 94 (1971) 377.

[15] J. Eberth et al., Nucl. Instr. and Meth. A 369 (1996) 135.

[16] J. Eberth et al., Prog. Part. Nucl. Phys. 38 (1997) 29.

[17] R.M. Lieder et al., Prog. Part. Nucl. Phys. 46 (2001) 399.

[18] R.M. Lieder et al., Nucl. Phys. A682 (2001) 279c.

[19] G.F. Knoll "Radiation Detection and Measurement". New York: Wiley and Sons. 4th. ed. (2010).

[20] P.W. Loscutoff and S.F. Bent, Annu. Rev. Phys. Chem. 57 (2006) 467.

[21] G. Maggioni et al., LNL Annual Report, (2011).

[22] Italian patent application RM2013A000379 (June 2013). 